\section{ANGINA PECTORIS: A CRITICISM AND A HYPOTHESIS.}

By E. H. COLBECK, M.D. Cantab., M.R.C.P. Lond,

PHYSICIAN TO OUT-PATIENTS AT THE CITY OF LONDON HOSPITAL FOR DISEASES OF THE CHEST; PHYSICIAN TO THE METROPOLITAN DISPENSARY.

THE field of cardiac pathology still resounds with the clash of conflicting arguments and the fray has nowhere been more hotly contested than over angina pectoris. Yet, in spite of the 61 attempts which, according to Huchard, have been made to elucidate this symptom group or syndrome, as it is now the fashion to call it, the precise method of its causation remains more or less a mystery. It will be the object of this paper to criticise the more important theories which have been advanced and also to submit a hypothesis whereby the mode of production of this curious and interesting group of symptoms is capable of explanation.

It is necessary, in view of the conflicting opinions held by clinicians with respect to the set or group of symptoms comprised under the term angina pectoris, to define clearly the nomenclature that will be adopted. If, as Professor T. Clifford Allbutt pertinently observers, the title angina pectoris be used to include a variety of symptom groups which, though they may present a superficial clinical resemblance in the manifestation of some form of cardiac perturbation, yet differ widely and fundamentally in their etiological and pathological relations, all definite use of names comes to an end. In this connexion, too, it may be pointed out that the use of such terms as "false" angina pectoris and "pseudo" angina pectoris is altogether objectionable and fallacious, inasmuch as it is obviously incorrect and unscientific to call a disease or group of symptoms by what it is not. rather than by what it is. For the profitable discussion of any scientific problem it is wel to deal so far as possible with clear-cut and definite con ceptions and with this object in view the group of symptoms included under angina pectoris will now be clearly defined.

The earliest and central feature of the paroxysm is pain, heart-rending, insistent, well-nigh insupportable pain, which though initially and mainly retro-sternal tends to radiate over the upper portion of the thorax and down one or both arms as far as the elbows or wrists, and sometimes as far as the fingers. A sense or apprehension of impending death is the more or less constant and dread accompaniment of the pain Agonised by this two-edged weapon the sufferer is rendered powerless and incapable of the slightest movement; he remains, as it were, petrified and rooted to the spot. The apprehension of sudden death is in the large majority of cases realised sooner or later, and post-mortem examination commonly reveals aortic and coronary atheroma associated, it may be, with a greater or less degree of myocardial degeneration.

Such, then, in outline is the clinical picture of angina pectoris, the picture drawn so forcibly and accurately by Heberden in the year 1768. Other so-called anginas are but mimicries of this terrible and deadly syndrome and in order to put an end to the misnomer and consequent nosological confusion involved in the use of the terms "false" angina pectoris, "pseudo" angina pectoris, and the like, I would suggest that the name angina cordis be applied to these allied but widely different cardiac perturbations. The term angina cordis, moreover, expresses more or less exactly the condition of the heart in these attacks and it can be suitably qualified in accordance with its mode of origin, as, for instance, angina cordis vasomotoria, angina cordis toxicata, \&c.

The association of angina pectoris with disease of the coronary arterits and myocardium was observed and insisted upon by Jenner and is now generally admitted.

The relation between these coronary and myocardial changes and the symptoms of angina pectoris has long been the subject of speculation, but it may safely be asserted that none of the theories hitherto advanced has afforded a perfectly satisfactory explanation. The theory of "intermittent claudication" which, according to Ösler, was originally advocated by Allan Burns, has been employed in the interpretation of the connexion between the coronary affection and the symptoms of angina pectoris. This interesting morbid condition is observed in the horse and occasionally in man and depends on arterial thrombosis or embolism whereby a large muscular territory, usually one or both limbs, is suddenly deprived of blood and becomes temporarily paralysed. The muscular paresis is apparently attended by great suffering and it is suggested that the pain and presumably the other phenomena of the anginal paroxysm are, in like manner, dependent on coronary ischæmia. It is, however, doubtful whether coronary thrombosis in man gives rise to pain and apart from this objection recurrent and frequently repeated attacks of angina which may occur without any appreciable disturbance or alteration in the action of the heart could not possibly be due to intermittent clandication.

The symptoms of angina pectoris and more particularly the pain that is so promment and characteristic a feature of the paroxysm have been ascribed by many distinguished authors to cramp of the cardiac muscle. The most extraordinary divergence of opinion appears with respect to this theory. Many eminent authorities deny that the cardiac muscle is capable of cramp, while other equally illustrious writers assert that this kind of painful muscular contraction can, and does, occur. From a physiological standpoint, however, there is no evidence that the heart is capable of the cramp-like contraction that is observed in skeletal muscles. And if the possibility of cramp of the heart be admitted its occurrence even when localised would surely intelfere with the orderly and uniform performance of the cardiac systole and in this way lead to much greater disturbance of the action of the heart and of the rhythm and character of the pulse than is commonly found to be the case. Furthermore, cramp of the heart does not account for the angor animi or sense of impending death which is a characteristic leature of the anginal attack. Why, too, does not cramp occur in connexion with disease of the rikht ventricle? Why, too, if cramp be concerned in the production of the symptoms of angina pectoris should the pain be relieved by the establishment of mitral regurgitation? Why, since the conditions which presumably favour the cccurrence of cramp are not infrequently fulfilled, should the phenomenon be so rarely ob:erved? Why should cramp so rarely affect the heart of the class of patients who come to hospitals? It is necessary to obtain a satisfactory reply to these questions before cramp of the heart be admitted as the cause of angina pectoris.

Current theories of angina pectoris link together cramp and paralysis of the cardiac muscle. But is not this blowing hot and cold with the same breath; are not these conditions the antithesis of each other, and mutual'y destructive from a causal point of view? Be this as it may the objections urged above to the agency of cramp apply equally forcibly to the operation of paralysis of the cardiac muscle as a cause of the anginal attack. Distension of the heart and stretching of the nerve elements in the cardiac wall cannot be a cause of the phenomena of angina if associated with increared arterial pressure, since the two conditions are impossible at the ame time. Moreover, dilatation and stretching of the cardiac walls are frequently observed and are not attended by the symptoms of angina pectoris.

The phenomena of the anginal attack bave been attributed by a large number of eminent authorities to neuralgia or neuritis of the cardiac nerves. The objections to this theory are that inflammatory and degenerative conditions of the cardiac nerves have frequently been observed without any of the symptoms of angina pectoris. Again, in those cases of angina in which nerve changes have been found there have also been observed well marked aortic and coronary atheroma, with or without myocardial degeneration. Neuralgia and neuritis of the cardiac nerves have also been found in association with symptoms which du not resemble those. of angina pectoris. The mode of onset of the anginal paroxysm and the rarity of its occurrence among women are features opposed to the purely nervous theory of its mode of production. Moreover, the angor animi is not explained by the theory under examination, nor does neuralgia of the cardiac nerves afford a satisfactory interpretation of the almost invariably fatal termination of angina pectoris.

Professor Clifford Allbutt is of the opinion that the pain of angina pectoris arises in the aorta and in cases of chronic disease of this vessel, at all events, is due to tensicn and stretching of the degenerated aortic walls. I cannot help thinking that for so able and brilliant a pleader Professor Allbutt's advocacy of this theory is somewhat half-hearted. 
The hypothesis in question does not permit the coronary and myocardial disease any causal influence in the production of the anginal attack. It is to be observed, however, that these changes in relation to the aortic disease are advantageous and conservative inasmuch as they make for lessened aortic blood pressure and therefore diminished aortic stress. A high blood pressure in the aorta of protracted duration is common enough without the incidence of anginal symptoms. Moreover, those occupations which are prone to give rise to aortic strain and stress do not furnish any large proportion of the cases of angina pectoris-indeed, angina pectoris is rarely observed among blacksmiths, labourers, navvies, porters, and the like. Nor, so far as I am aware, does this hypothesis attempt to explain the sense of impending death which, as I have already insisted, is one of the central features of the anginal seizure. It seems to me that Professor Allbutt is much nearer the mark when he suggests that in addition to the static changes which are found there is a dynamical factor in the causation of this symptom group.

The vaso-motor phenomena that are observed in connexion with angina pectoris will be considered later. A hypothesis which aspires to elucidate the mode of production of the symptoms of angina pectoris must account not only for the dolor pectoris but also for the angor animi. It must likewise afford a satisfactory interpretation of the character of the pulse and of the vaso-motor phenomena associated with the paroxysm. The various theories which have been under review fail to satisfy fully these requirements and herein lies the justification, if one be needed, for yet another attempt to explain the mode of production of this remarkable and enigmatical syndrome. It is now generally allowed that disease of the coronary arteries and of the myocardium is in some way associated causally with the incidence of angina pectoris. The coronary atheroma, which is the morbid condition usually found, and the myocardial degeneration are unequally distributed over the wall of the heart. Furthermore, careful microscopical examination usually reveals a greater degree and extent of myocardial disease than the macroscopical appearances indicate. It is probable, too, that the nervous tissue-i.e., ganglia and nerve fibres-distributed over the wall of the heart suffers in common with the muscular substance of the organ and tends therefore to undergo similar degenerative changes. I would again insist on the fact that in both cases the morbid changes have a "patchy" irregular disposition.

So long as the heart is acting quietly the areas of degenerated muscular tissue are able, even with a relatively insufficient blood-supply, to take their share in resisting and overcoming the intra-cardiac pressure during systole. So soon, however, as the work of the heart is increased, as, for instance, by exertion, emotion, \&c:; the area or areas of degenerated muscular wall are at a double disadvantage, inasmuch as the diseased and partially occluded coronary vessels are unable to satisfy the increased demand for blood to the territories or patches of deteriorated muscular tissue. The areas of degenerated ventricular wall are thus unable to take their proper share in resisting the increased intra-cardiac pressure and in consequence undergo more or less distension and stretching. Herein lies the explanation of the pain experienced during the anginal seizure, since the affected portions of the ventricular wall contain nervous tissue in an unduly impressionable and irritable condition, which must be subjected to a considerable degree of stretching and tension. In other words, the hypothesis I suggest ascribes the pain experienced in the anginal paroxysm to localised distension and stretching of the ventricular wall. If this hypothesis be well founded it must also satisfy the requirements that were detailed when the question of cramp of the cardiac muscle was discussed and I think this will on examination be found to be the case.

The hypothesis does not entail any interference with the regular and uniform performance of the ventricular systole the wave of contraction passes uninterruptedly from apex to base, but over certain portions of the ventricular wall it is qualitatively and quantitatively deficient. In these con ditions the action of the heart and the rhythm and character of the pulse may remain, so far as physical examination is concerned, undisturbed and unaltered, and in the majority of anginal seizures this state of affairs obtains. It is conceivable, however, that should the area or areas of degenerated ventricular wall exceed a certain proportion in relation to the amount of sound muscular tissue the heart might falter, the pulse become irregular, and the blood pressure fall. This sequence of events has also been observed, more especially in old-standing cases and in the terminal stages of frequently repeated anginal attacks.

The establishment of mitral regurgitation in the course of angina pectoris relieves the intra-ventricular pressure and in this way diminishes the stress on the cardiac wall, and hence the degree of distension and stretching of the diseased muscular territories. Nitrites and other vaso-dilators owe their beneficial influence to a similar mode of action-viz., the reduction of intra-ventricular pressure. The safety-valve action at the tricuspid opening effectually precludes the operation of the conditions which might otherwise give rise to undue stress on the wall of the right ventricle and it is for this reason, I take it, that anginal phenomena are rarely if ever associated with disease on this side of the heart. Again, the hypothesis which I suggest provides a feasible and attractive explanation of the angor animi which is usually more or less neglected in the current theories of the pathogenesis of angina pectoris. It is obvious that if distension and stretching of localised areas of the cardiac wall take place during systole the ventricular parietes are simultaneously undergoing contraction and expansion. The effect of this dynamical perversion of the cardiac movements on the impressions transmitted to the cardiac centre during the ventricular contraction must be contradictory and antagonistic, and the intensity of the opposed efferent impulses will be influenced by the relative amounts of sound and degenerated muscular tissue and also by the degree of extra work demanded of the heart. The cardiac centre must in these circumstances be thrown into a turmoil, and it is hardly a matter for surprise that a threatened disorganisation of the very citadel of life should occasion the sensation of impending death. Moreover, the perturbation of the cardiac centre spreads, no doubt, to adjacent centres and herein lies the explanation of the vasomotor phenomena as well as of the nausea, vomiting, flatulence, hiccough, and so forth which so commonly accompany the anginal paroxysm.

A few subsidiary and contingent conditions still remain for consideration. The extraordinary variability in the incidence of anginal attacks is explicable on the assumption that the coronary and myocardial affection does not always provide the exact conditions for the production of localised stress on the ventricular wall. Indeed, it is not unreasonable to surmise in the light of the present hypothesis that the production of an anginal seizure in coronary and myocardial disease is always more or less of an accident. Clinical observations appear to bear out this supposition. The occurrence of sudden death can be explained in two ways. Thus the sudden arrival at the cardiac centre of equally balanced antagonistic impulses might inhibit the action of the centre, or that portion of the ventricular wall which is known as Kronecker's centre in the dog and is situated in the lower portion of the upper third of the inter-ventricular septum may be the seat of disease (coronary or parietal) and hence of distension and stretching in the manner previously indicated, so that sudden death may be due either to a reflex or to a local cause.

The remarkable immunity from anginal symptoms that is displayed by the class of people who seek advice and treatment at hospitals is, I imagine, due to the fact that the conditions of life in which these patients live lead to more general and extensive myocardial degeneration and hence to more readily produced failure of the heart than is the case with the more well-to-do members of the community. Angina sine dolore is explained on the assumption that the deterioration of the nervous elements in the cardiac wall becomes so great that they fail to react to stimuli which ordinarily give rise to the sensation of pain. It is extremely interesting to observe that, under the operation of the causal factors I suggest, the incidence of this form of angina pectoris justifies a serious prognosis, since it implies grave deterioration of the wall of the heart.

The relation between "pseudo" angina pectoris, or angina cordis vasomotoria as I prefer to call it, and angina pectoris still remains to be discussed. In angina cordis vasomotoria the essential feature of the paroxysm is, as Solis-Cohen has pointed out, a vaso-motor ataxia, whereby the heart is suddenly exposed to stress which, after a period of stormy nervous action, subsides and enables the organ again to obtain control of the circulation. The cardiac embarrassment, in these conditions, is due to sudden and general arterial constriction, which in turn depends on the operation of imperfectly controlled central and peripheral stimuli on unstable and unduly sensitive vaso-motor and nervous centres. 
In angina pectoris, on the other hand, although the general arterial blood pressure is probably always above the normal, the vaso-motor phenomena are, in part at least, secondary and not primary features of the attack. It is possible, indeed not improbable, that spasm of the coronary arteries (which are affected in common with the rest of the arterial system) plays a similar but subordinate rôle in the production of cardiac pain in angina cordis vasomotoria to that which organic disease of these vessels does in the causation of an attack of angina pectoris. Moreover, it is conceivable that long-continued and frequently repeated attacks of angina cordis from any cause might nltimately lead to disease of the coronary arteries and myocardium and in this way the various forms of angina may merge imperceptibly into one another. From this point of view, then, the existing antagonism may be resolved into a harmonious whole by means of which the incidence of cardiac angina is freed from the difficulties which have hitherto beset the solution of its occurrence and mode of production.

Upper Berkeley-street, $\mathrm{W}$.

\section{A CLIMATOLOGICAL ASPECT OF THE NAUHEIM TREATMENT OF DISEASES OF THE HEART.}

By LESLIE C. THORNE THORNE, M. D., B.SC. DURH., MEDICAL EXAMINER TO THE TECHNICAL EDUCATION BOABD OF THE LONDON COUTTY COUNCIL.

IT is now many years since the Nauheim treatment of diseases of the heart was first practised in England, and those who have devoted special attention to the subject have had time to observe the effects of the English climate upon it and have been able so to regulate its application as to produce the best results obtainable, these results being in many cases of a highly satisfactory nature. There are still, however, a large number of medical men who either do not use this method of treatment at all or do not give it that place of importance as a therapeutic agent which it rightly deserves. The reason for this, I believe, lies in the fact that the Nauheim treatment when administered in England bas in some ways to be modified and adapted to the climate, whereas many medical men who have given it what they believe to be a fair trial have prescribed the comparatively long and frequent baths and the frequent resistance exercises that often produce good results at Nauheim, but, according to my experience in London, only benefit a small minority of mild cases of heart disease and do definite harm to the more serious cases.

As I am convinced that many who have hitherto found this treatment of little use in England would be led to regard it as a really valuable therapeutic agent were they to adopt the methods of administration that $I$ have found most successful in London, I do not hesitate to give a short sketch of a course as I have carried it out for some years with very satisfactory results, especially in cases which have not been greatly benefited by rest, the administration of drugs, or other methods of treatment. In giving this sketch I shall endeavour to mention all the really important points to be attended to, su that any medical man who has not hitherto administered the Nauheim treatment may be able to do so by carefully carrying out the directions given.

The administration of the baths - Taking, for example, a case of dilated irritable heart, a sequel of influenza, causing symptoms of vertigo, palpitation, dy spnea, insomnia, cardiac pain, lassitude, and general invalidism, I begin by giving a three or four minutes' bath at a temperature of $97^{\circ}$ or $98^{\circ} \mathrm{F}$., containing from four to five pounds of Droitwich salt and from five to six ounces of calcium chloride. The pulse is carefully noted before, during, and after the bath and the result aimed at, and usually obtained, is a diminution in frequency, an increase in volume, and a lowering of tension. The patient goes to bed for one hour after the bath and is not allowed during this time to read, write, or talk. A bath of similar strength, temperature, and duration is given the following day. On the third day the Schott exercises are usually given instead of a bath, the patient resting for a minute or so between each exercise and lying down for half an hour after them.

Firequency of baths.--The baths are given on two consecutive days and the exercises on the third day for the first week or fortnight, according to the severity of the case and the result produced upon the heart's action. After the first week or fortnigbt baths are given three days in succession with an interval for exercises on the fourth day. In my opinion it is a mistake to give baths for more than three days in succession even towards the end of the course.

Strength of baths. - If the patient does well and the circulatory system reacts well I increase the strength of the baths by one pound of sodium chloride and one ounce of calcium chloride every second bath up to the tenth or twelfth. To the tenth or twelfth bath $I$ add half of one of Sandow's effervescing packets, at the same time reducing the sodium chloride by about two pounds and the calcium chloride by about two ounces. If this change proves satisfactory $I$ again start, raising the strength of the baths as in the first instance, increasing the effervescence to threequarters of a packet at about the sixteenth bath, and a whole packet at the twentieth. The number of baths taken in a course varies from about 25 to 30 , any greater number being, in my experience, likely to weaken the patient and to decrease rather than to increase the good that has been done. By the end of the course the patient is having a bath containing from 12 to 14 pounds of sodium chloride, from 14 to 16 ounces of calcium chloride, and one boxful of Sandow's effervescing tablets.

Duration of immersion. - As I have previously said, I begin by giving a three to four minutes' bath and with an average case I find that the best plan is to lengthen the time of immersion by one minute every second bath, curtailing it, however, by two or three minutes when the first effervescing bath is giver.

Temperature of baths. - The first bath is given at a temperature of $97^{\circ}$ or $98^{\circ}$, this temperature being lowered $1^{\circ}$ every second bath down to $94^{\circ}$ or even $93^{\circ}$ in exceptional cases, but it is often found that a good reaction cannot be obtained with a temperature below $96^{\circ}$ and in these cases that temperature is adhered to throughout the course. At Nauheim, where the treatment is only given from the beginning of May to the end of September, baths of a lower temperature may have some advantages, but it is my experience that a good reaction is not obtained from a bath given at a lower temperature than $93^{\circ}$.

The administration of the exercises. - The exercises should be given by as experienced an operator as possible and by one who has been trained to watch the pulse and to regulate the resistance given by the effect produced. If increased arterial tension and rapidity of pulse are produced, then the exercises are being given too forcibly and too rapidly and are doing harm rather than good. As a rule, if the exercises are properly given, they rarely fail to lower the rate and tension of the pulse and to increase its volume. I only give the exercises on the days when there is no bath taken, as I believe this is the wisest plan to pursue all through the course. I am certainly convinced that to give both bath and exercises on the same day for a longer period than the last week of the treatment is unwise and I am doubtful whether even this is advisable except in cases of very slight cardiac trouble. The exercises are by no means as powerful an agent as the baths and if no experienced operator is procurable it is best to omit them entirely.

The general care of a case during treatment is of great importance : no definite rules can be laid down which would be suitable to all, but it is essential that the patient should absolutely give up business and social engagements and should, if well enough, take a prescribed amount of walking exercise both morning and afternoon and have the digestion and bowels well attended to. That these general directions carefully carried out help to a satisfactory result is manifest, but that they are not in themselves enough to produce that result I have proved, as I have often given cases general treatment for some time before using the Nauheim baths and have failed to obtain improvement as rapid, satisfactory, or lasting as that resulting from a course of baths and exercises.

I have not entered here into a discussion with regard to the forms of cardiac disease most suitable for treatment, as that is not within the scope of this paper, but in advising the treatment and giving a prognosis as to the result it must always be remembered that an organically diseased heart cannot be converted into a sound organ by this or any other method of treatment, but that a dilated and enfeebled heart with flabby muscular walls and imperfect contractile powers can be cured by this method in a way that, in my experience, is not possible by any other form of treatment. the reason for this being that whereas digitalis and other allied cardiac 